Jan Kolář, Department of Mathematical Analysis, Charles University, Sokolovská 83, 18675 Praha 8, Czech Republic.

e-mail: kolar@karlin.mff.cuni.cz

\title{
SIMULTANEOUS EXTENSION OPERATORS FOR THE DENSITY TOPOLOGY
}

\begin{abstract}
Given a set $M \subset \mathbb{R}$ of Lebesgue measure zero, let $\left.\mathcal{B}_{1}\right|_{M}$ be the set of all restrictions to $M$ of bounded Baire one functions on $\mathbb{R}$ and $\mathcal{A}$ the set of all bounded approximately continuous functions on $\mathbb{R}$. We discuss the existence of simultaneous extension operators for $\left.\mathcal{B}_{1}\right|_{M}$ and $\mathcal{A}$. We show that there exists a positive linear operator $L:\left.\mathcal{B}_{1}\right|_{M} \rightarrow \mathcal{A}$ such that $\left.L(g)\right|_{M}=g$ for all $\left.g \in \mathcal{B}_{1}\right|_{M}$, if and only if $M$ is a scattered set and this is the case if and only if there exists a continuous linear operator $L_{1}:\left.\mathcal{B}_{1}\right|_{M} \rightarrow \mathcal{A}$ with the same property. Also, we show that there exist non-regular continuous linear operators $T_{1}: \ell_{\infty} \rightarrow \mathcal{A}$ and $T_{2}: \mathcal{A} \rightarrow \mathcal{A}$.
\end{abstract}

Let $\lambda$ denote Lebesgue measure on $\mathbb{R}$. Recall that $x \in \mathbb{R}$ is a point of density of a Lebesgue measurable set $M \subset \mathbb{R}$ if $\lim _{h \backslash 0} \lambda(M \cap(x-h, x+h)) / 2 h=1$. A point $x$ is a dispersion point of $M$ if $\lim _{h \backslash 0} \lambda(M \cap(x-h, x+h)) / 2 h=0$. A measurable set $M$ is said to be density open if every point $x \in M$ is a point of density of $M$. The density open sets form a topology $d$ on $\mathbb{R}$, called the density topology. It turns out that the so called approximately continuous functions are exactly the continuous functions in the density topology.

By $\mathcal{B}_{1}$ we denote the set of all bounded Baire one functions on $\mathbb{R}$ and by $\mathcal{A}$ the set of all bounded approximately continuous functions on $\mathbb{R}$. Note that $\mathcal{A} \subset \mathcal{B}_{1}$ [LMZ, 6.8.b]. For $M \subset \mathbb{R},\left.\mathcal{B}_{1}\right|_{M}$ is the space of restrictions to $M$ of all functions $f \in \mathcal{B}_{1} . \mathcal{A}, \mathcal{B}_{1}$ and $\left.\mathcal{B}_{1}\right|_{M}$ are Banach spaces equipped with the norm $\|f\|=\sup _{x}|f(x)|$. By $\chi_{M}$ we denote the characteristic function of the set $M$, and $\chi_{a}=\chi_{\{a\}}$ for $a \in \mathbb{R}$. If $L$ is an operator defined on $\left.\mathcal{B}_{1}\right|_{M}$ and $f \in \mathcal{B}_{1}$, we

Key Words: simultaneous extension operator, approximately continuous functions, density topology

Mathematical Reviews subject classification: 54C20, 46E15, 54A10

Received by the editors July 22, 1998

*Support of the Charles University Grant Agency (GAUK 165/99), DAAD and Stiftung "Universität und Gesellschaft" (Universität Konstanz) is gratefully acknowledged. 
often write $L(f)$ instead of $L\left(\left.f\right|_{M}\right)$. By an operator we always mean a linear operator. If $L$ is an operator and $L(f)$ is an extension of $f$ for every function $f$ in the domain of $L$, we say that $L$ is a simultaneous extension operator.

Let $M \subset \mathbb{R}$ be a set of Lebesgue measure zero and $\left.f \in \mathcal{B}_{1}\right|_{M}$. By the Theorem of Petruska and Laczkovich ([PL, 3.2(iii)], see also [LMZ, 8.3]), there is a bounded approximately continuous function $F_{f}$ on $\mathbb{R}$ which extends $f$. Can $F_{f}$ be assigned in a nice way; i.e., does there exist a simultaneous extension operator $L: f \mapsto F_{f}$ which is positive or continuous? This question is inspired by Borsuk's simultaneous extension theorem which has been generalized in many different ways. We present here the following variant which is due to G. J. O. Jameson $[\mathrm{J}]$. By $\mathcal{C}_{b}(Y)$ we denote the set of all bounded continuous functions on a set $Y$.

Theorem (Jameson). Let $S$ be a topological space, $A$ be a closed subset of $S$ such that every function $f \in \mathcal{C}_{b}(A)$ can be extended continuously to $S$. Let $X$ be a separable linear subspace of $\mathcal{C}_{b}(A)$. Then there is a positive, isometric simultaneous extension operator $L: X \rightarrow \mathcal{C}_{b}(S)$. In particular, if $A$ is compact and metrizable, then such an operator can be defined on the whole of $\mathcal{C}_{b}(A)$.

In this paper we show that, in the case of approximately continuous functions, a positive and/or isometric simultaneous extension operator exists if and only if the set $M$ is scattered.

A set $M \subset \mathbb{R}$ is called scattered if it contains no dense in itself non-empty subset $[\mathrm{K}, 1.9 . \mathrm{VI}]$.

Proposition 1. Let $M \subset \mathbb{R}$ and $L:\left.\mathcal{B}_{1}\right|_{M} \rightarrow \mathcal{A}$ be a simultaneous extension operator. If $L$ is positive or continuous, then $M$ is scattered.

Proof. The case of continuous operator $L$ is more complicated to deal with. It will be convenient to index symbols by points of the set $M$. The first part of the proof is common for both cases.

Assume that $M$ is not scattered. Let $A \neq \emptyset$ be a dense in itself subset of $M$. Let $a_{0} \in A$ and $I_{0}=J_{0}$ be an open interval containing $a_{0}$. If $I_{0} \supset$ $J_{0} \supset \cdots \supset I_{n-1} \supset J_{n-1}$ and $a_{0}, \ldots, a_{n-1} \in A$ have already been defined, find $a_{n} \in A \cap J_{n-1} \backslash\left\{a_{0}, \ldots, a_{n-1}\right\}$. If $L$ is positive, put

$$
G_{a_{n}}=G_{n}:=\left\{y \in \mathbb{R}: L\left(\chi_{a_{n}}\right)(y)>\frac{1}{2}\right\} .
$$

If $L$ is continuous, put

$G_{a_{n}}=G_{n}:=\left\{y \in \mathbb{R}: L\left(\chi_{a_{n}}+\sum_{i \in D} \chi_{a_{i}}\right)(y)>\frac{1}{2}\right.$ for every $\left.D \subset\{1, \ldots, n-1\}\right\}$. 
Since $L\left(\chi_{a_{i}}\right)$ are approximately continuous, $L\left(\chi_{a_{n}}\right)\left(a_{n}\right)=1$, and $L\left(\chi_{a_{i}}\right)\left(a_{n}\right)=$ 0 for $i=1, \ldots, n-1$, the point $a_{n}$ is a point of density of the set $G_{n}$. Hence there is $h_{a_{n}}=h_{n} \in\left(0, \frac{1}{n}\right)$ such that $I_{a_{n}}=I_{n}:=\left(a_{n}-h_{n}, a_{n}+h_{n}\right) \subset J_{n-1}$ and $^{1} \lambda\left(G_{n} \cap I_{n}\right)>\left(1-2^{-n}\right) 2 h_{n}$. Let $J_{n}:=\left(a_{n}-2^{-n} h_{n}, a_{n}+2^{-n} h_{n}\right)$.

Let $\{x\}=\bigcap_{n=1}^{\infty} \overline{I_{n}}=\bigcap_{n=1}^{\infty} \overline{J_{n}}$. Note that $a_{n} \rightarrow x$ and, for every $n$,

$$
\lambda\left(G_{n} \cap\left(x-h_{n}, x+h_{n}\right)\right) \geq \lambda\left(G_{n} \cap I_{n}\right)-2^{-n} h_{n}>\left(1-2^{-n+1}\right) 2 h_{n} .
$$

If $L$ is positive, $\left\{n_{m}\right\}$ is an arbitrary increasing sequence, $b_{m}:=a_{n_{m}}$ and $H_{m}:=G_{n_{m}}$, then $L\left(\sum_{m=1}^{\infty} \chi_{b_{m}}\right)>\frac{1}{2}$ on the set $\bigcup_{m} H_{m}$. (It is easy to see that $\sum \chi_{b_{m}}$ is a Baire one function since $b_{m} \rightarrow x$.) Furthermore, for every $m$, $\lambda\left(\left(\bigcup_{k} H_{k}\right) \cap\left(x-h_{n_{m}}, x+h_{n_{m}}\right)\right) / 2 h_{n_{m}}>1-2^{-n_{m}+1}$ and $h_{n_{m}} \rightarrow 0$. Hence $x$ is not a point of density of $\mathbb{R} \backslash \bigcup_{m} H_{m}$. By our assumptions and because $\sum_{m=1}^{\infty} \chi_{b_{m}} \in \mathcal{B}_{1}$ we have that $L\left(\sum_{m=1}^{\infty} \chi_{b_{m}}\right)$ is approximately continuous; so $L\left(\sum_{m=1}^{\infty} \chi_{b_{m}}\right)(x) \geq \frac{1}{2}$. For $i, m \in \mathbb{N}$ put $n_{m}^{(i)}=2^{i}(2 m-1)$. Then $\left\{n_{m}^{(i)}\right\}_{m=1}^{\infty}$ $(i=1,2 \ldots)$ are disjoint increasing sequences. By the linearity and positivity of $L$,

$$
L\left(\sum_{n=1}^{\infty} \chi_{a_{n}}\right)(x) \geq \sum_{i=1}^{N} L\left(\sum_{m=1}^{\infty} \chi_{a_{n_{m}^{(i)}}}\right)(x) \geq \frac{1}{2} N \rightarrow \infty \quad(\text { as } N \rightarrow \infty),
$$

which is a contradiction.

If $L$ is continuous, let $K \in \mathbb{N}$ be such that $\left.f \in \mathcal{B}_{1}\right|_{M}$ and $\|f\| \leq 1$ implies $\|L(f)\| \leq K$. Let $a_{i}^{(1)}:=a_{i}$ for $i \in \mathbb{N}$. Proceed by induction. Assume $n \in \mathbb{N}$ is fixed and $\left\{a_{i}^{(n-1)}\right\}_{i=1}^{\infty}$ is already defined. For $k=1,2, \ldots, 4 K$ put

$$
A_{n-1}^{k}:=\bigcap\left\{y \in I_{a_{n-1}^{(n-1)}}: L\left(\sum_{i} \chi_{b_{i}}\right)(y) \geq-\frac{1}{4}\right\}
$$

where the intersection is taken over all subsequences $\left\{b_{i}\right\}_{i=1}^{\infty}$ of $\left\{a_{4 K i+k}^{(n-1)}\right\}_{i=1}^{\infty}$. Every set $A_{n-1}^{k}$ is measurable since it is an intersection of closed sets in the density topology. Hence closed itself. Furthermore $\bigcup_{k=1}^{4 K} A_{n-1}^{k}=I_{a_{n-1}^{(n-1)}}$. Indeed, if $y \in I_{a_{n-1}^{(n-1)}}$ does not belong to $A_{n-1}^{k}$ for any $k=1,2, \ldots, 4 K$, then there is a subsequence $\left\{b_{i}\right\}_{i=1}^{\infty}$ of $\left\{a_{i}^{(n-1)}\right\}_{i=1}^{\infty}$ such that $L\left(\sum_{i} \chi_{b_{i}}\right)(y)<$ $4 K \cdot\left(-\frac{1}{4}\right)$ which is contrary to the definition of $K$. Hence there exists $k \in$ $\{1,2, \ldots, 4 K\}$ such that $\lambda\left(A_{n-1}^{k}\right) \geq \lambda\left(I_{a_{n-1}^{(n-1)}}\right) / 4 K$. For such $k$, let $a_{i}^{(n)}:=$ $a_{4 K i+k}^{(n-1)}$ and $A_{n-1}:=A_{n-1}^{k}$.

\footnotetext{
${ }^{1}$ For our purposes it is enough to have $\lambda\left(G_{n} \cap I_{n}\right)>c \cdot 2 h_{n}$, where $c \in(0,1)$ is a constant depending on the norm of $L$, or, say, $c=\frac{1}{2}$ in the case $L$ is positive.
} 
Let $\widetilde{a}_{n}:=a_{n}^{(n)}$ be the diagonal sequence. Let $\left\{n_{m}\right\}$ be an increasing sequence, $b_{m}:=\widetilde{a}_{n_{m}}$. Fix $m_{0} \in \mathbb{N}$. Then ${ }^{2}$

$$
\left\{b_{m}\right\}_{m=m_{0}+1}^{\infty} \subset\left\{a_{i}^{(i)}\right\}_{i=n_{\left(m_{0}+1\right)}}^{\infty} \subset\left\{a_{i}^{(i)}\right\}_{i=n_{m_{0}}+1}^{\infty} \subset\left\{a_{i}^{\left(n_{m_{0}}+1\right)}\right\}_{i=n_{m_{0}}+1}^{\infty}
$$

and $L\left(\sum_{m>m_{0}} \chi_{b_{m}}\right) \geq-\frac{1}{4}$ on $A_{n_{m_{0}}} \subset I_{b_{m_{0}}}$. Recall that by definition of $G_{b_{m_{0}}}$ $L\left(\sum_{m \leq m_{0}} \chi_{b_{m}}\right)>\frac{1}{2}$ on $G_{b_{m_{0}}}$. Thus $L\left(\sum_{m=1}^{\infty} \chi_{b_{m}}\right) \geq \frac{1}{4}$ on $A_{n_{m_{0}}} \cap G_{b_{m_{0}}} \subset I_{b_{m_{0}}}$ which has measure at least $\lambda\left(A_{n_{m_{0}}}\right)-\lambda\left(I_{b_{m_{0}}} \backslash G_{b_{m_{0}}}\right) \geq\left(\frac{1}{4 K}-2^{-n_{m_{0}}}\right) 2 h_{b_{m_{0}}}$. This implies

$\lambda\left(A_{n_{m_{0}}} \cap G_{b_{m_{0}}} \cap\left(x-h_{b_{m_{0}}}, x+h_{b_{m_{0}}}\right)\right) \geq\left(\frac{1}{4 K}-2^{-n_{m_{0}}}-2^{-n_{m_{0}}}\right) 2 h_{b_{m_{0}}} \geq \frac{1}{8 K} 2 h_{b_{m_{0}}}$

if $m_{0}$ is large enough. Hence $L\left(\sum_{m=1}^{\infty} \chi_{b_{m}}(x)\right) \geq \frac{1}{4}$ by approximate continuity. For $i, m \in \mathbb{N}$ put $n_{m}^{(i)}=2^{i}(2 m-1)$. Then $\left\{n_{m}^{(i)}\right\}_{m=1}^{\infty}(i=1,2 \ldots)$ are disjoint

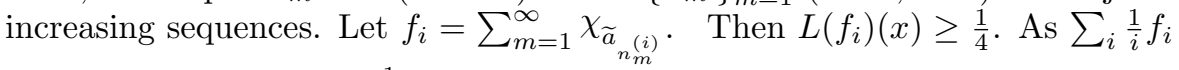
converges uniformly, $\sum_{i} \frac{1}{i} f_{i} \in \mathcal{B}_{1}$ and we obtain by the continuity of $L$ that

$$
L\left(\sum_{i} \frac{1}{i} f_{i}\right)(x)=\lim _{N \rightarrow \infty} L\left(\sum_{i=1}^{N} \frac{1}{i} f_{i}\right)(x) \geq \lim _{N \rightarrow \infty} \frac{1}{4} \sum_{i=1}^{N} \frac{1}{i}=\infty .
$$

This is a contradiction for the case of continuous extension operator.

Recall that a family $\left\{A_{\iota}\right\}_{\iota \in \mathcal{I}}$ of subsets of a topological space $(X, \tau)$ is discrete if for every $x \in X$ there is a $\tau$-neighborhood $U$ of $x$ such that $U$ intersects $A_{\iota}$ for at most one $\iota \in \mathcal{I}$. Note that every discrete family is disjoint.

A subset $M$ of $(X, \tau)$ is called strongly discrete if there exists a family $\left\{G_{x}\right\}_{x \in M}$ of $\tau$-open subsets of $X$, discrete in $(X, \tau)$, such that $x \in G_{x}$ for every $x \in M$. (However, some authors give a non-equivalent definition replacing "discrete in $(X, \tau)$ " by "disjoint".)

Lemma 2. Let $M$ be a scattered subset of $\mathbb{R}$. Then $M$ is a strongly discrete subset of $(\mathbb{R}, d)$.

Proof. (This simpler proof was suggested by L. Zajičcek.) We know that $M$ is countable $([\mathrm{K}, 2.23 . \mathrm{V}])$ and $\mathrm{G}_{\delta}([\mathrm{K}, 2.24 . \mathrm{III} .1 \mathrm{a}])$. Let $x_{n}, n \in I \subset \mathbb{N}$, be the points of $M$. Let $f: M \rightarrow \mathbb{R}$ be defined by $f\left(x_{n}\right)=n$ for $n \in I$ and $f(x)=0$ for $x \in \mathbb{R} \backslash M$. Then $f$ is obviously $\mathrm{F}_{\sigma}$-measurable; i.e., $f \in \mathcal{B}_{1}$. By the unbounded version of the Theorem of Petruska and Laczkovich ([PL, 3.2(iii)]), $\left.f\right|_{M}$ can be extended to an approximately continuous function $g$ on

\footnotetext{
${ }^{2}$ We write $\left\{c_{m}\right\} \subset\left\{d_{m}\right\}$ if sequence $\left\{c_{m}\right\}$ is a subsequence of $\left\{d_{m}\right\}$.
} 
$\mathbb{R}$. For $n \in I$ and $x=x_{n}$ let $G_{x}=g^{-1}((n-1 / 3, n+1 / 3))$. Then $x \in G_{x}$ and $\left\{G_{x}\right\}_{x \in M}$ is a family of density open subsets, discrete in the density topology.

Lemma 3. Let $M \subset \mathbb{R}$. Let $\left\{G_{x}\right\}_{x \in M}$ be a family of density open subsets of $\mathbb{R}$, discrete in $(\mathbb{R}, d)$, such that $x \in G_{x}$ for every $x \in M$. Then there exists a family $\left\{f_{x}\right\}_{x \in M}$ of approximately continuous functions $f_{x}: \mathbb{R} \rightarrow[0,1]$, such that $\left.f_{x}\right|_{M}=\left.\chi_{\{x\}}\right|_{M}$ for every $x \in M,\left\{y: f_{x_{1}}(y) \neq 0\right\} \cap\left\{y: f_{x_{2}}(y) \neq 0\right\}=\emptyset$ whenever $x_{1} \neq x_{2}, x_{1}, x_{2} \in M$, and for arbitrary function $g: M \rightarrow \mathbb{R}$, the function $F_{g}(y)=\sum_{x \in M} g(x) f_{x}(y)$ is approximately continuous and $\left.F_{g}\right|_{M}=g$.

Proof. By complete regularity of the density topology (cf. [LMZ, 6.9]), for each $x \in M$ there is an approximately continuous function $f_{x}: \mathbb{R} \rightarrow[0,1]$, such that $f_{x}(x)=1$ and $f_{x}(y)=0$ for $y \in \mathbb{R} \backslash G_{x}$. Thus $\left.f_{x}\right|_{M}=\chi_{\{x\}} \mid M$. Since the family $\left\{G_{x}\right\}_{x \in M}$ is discrete in the density topology, the function $F_{g}$ is approximately continuous for an arbitrary function $g$ on $M$.

Remark 3.1. By the Lusin-Menchoff property of the density topology (cf. [LMZ, 6.9.g]), there exist density open sets $H_{x},(x \in M), x \in H_{x} \subset \bar{H}_{x} \subset G_{x}$. Using complete regularity for each pair $\left(x, H_{x}\right)$ we obtain $f_{x}$ such that the family of closed supports of functions $f_{x}$ is discrete in the density topology.

Theorem 4. For a set $M \subset \mathbb{R}$, the following conditions are equivalent:

(i) $M$ is scattered,

(ii) $M$ is a strongly discrete subset of $(\mathbb{R}, d)$,

(iii) there exists a positive simultaneous extension operator $L:\left.\mathcal{B}_{1}\right|_{M} \rightarrow \mathcal{A}$,

(iv) there exists a continuous simultaneous extension operator $L:\left.\mathcal{B}_{1}\right|_{M} \rightarrow \mathcal{A}$,

(v) there exists a positive isometric simultaneous extension operator $L:\left.\mathcal{B}_{1}\right|_{M}$ $\rightarrow \mathcal{A}$.

Proof. By Lemma 2, (i) implies (ii) and Lemma 3 shows that (ii) implies (iii), (iv), (v). The implications (iii) $\Longrightarrow$ (i), (iv) $\Longrightarrow$ (i) and (v) $\Longrightarrow$ (i) follow from Proposition 1.

Remarks 4.1. 1. Notice that we did not need to restrict ourselves to bounded functions. $\mathcal{A}$ could be the space of all approximately continuous functions on $\mathbb{R}$ and $\left.\mathcal{B}_{1}\right|_{M}$ the space of all restrictions (to $M$ ) of Baire one functions. All results of this paper hold in this setting as well. (The continuity of $L$ is to be understood as continuity in the topology of uniform convergence). 
2. The proof of Proposition 1 cannot be based on the assertion that $\mathcal{A} \subset \mathcal{B}_{1}$ only but must use other properties of approximately continuous functions. Indeed, if $M \subset \mathbb{R}$ is an ambivalent set (both $\mathrm{F}_{\sigma}$ and $\mathrm{G}_{\delta}$ ), then there exists a positive isometric simultaneous extension operator $L:\left.\mathcal{B}_{1}\right|_{M} \rightarrow \mathcal{B}_{1}$ (for example given by the formula $L\left(\left.f\right|_{M}\right):=f \cdot \chi_{M}$ for $f \in B_{1}$; note that $\left.\chi_{M} \in B_{1}\right)$.

Since the proof of Proposition 1 is much more complicated in the case of continuous simultaneous extension operator $L$, the following idea arose which was inspired by a well developed theory of operators on Banach lattices: Could we prove first that $L$ is regular, thus reducing to the case of positive simultaneous extension operator? We will see that this idea fails.

Recall that, for $E, F$ Banach lattices, we say that a continuous operator $T: E \rightarrow F$ is regular if it can be decomposed as $T=P_{1}-P_{2}$ where $P_{1}, P_{2}$ are positive and continuous. In $[\mathrm{X}]$, the question is discussed, whether every continuous operator $E \rightarrow F$ is regular, for cases in which each $E$ and $F$ is one of Banach lattices $\ell_{p}(1 \leq p<\infty), \ell_{\infty}, c_{0}, c, \mathcal{C}[0,1]$ and $\mathcal{C}(K)$.

Example 5. There exists a continuous operator $T_{1}: \ell_{\infty} \rightarrow \mathcal{A}$ which is not regular.

Proof. Let $r_{n}(x)=\operatorname{sgn} \sin (2 \pi n x)$ be the Rademacher's functions on $[0,1]$. They form an orthonormal set in $L_{2}[0,1]$ and consequently $\left\|\sum_{n=1}^{m} a_{n} r_{n}\right\|_{L_{2}}=$ $\left\|\left\{a_{n}\right\}_{n=1}^{m}\right\|_{\ell_{2}}$. In particular, for $t>0$

$$
\begin{aligned}
& \lambda\left(\left\{x \in[0,1]:\left|\frac{1}{m} \sum_{n=1}^{m} a_{n} r_{n}(x)\right|>t\right\}\right) \leq\left\|\frac{1}{t m} \sum_{n=1}^{m} a_{n} r_{n}\right\|_{L_{2}}^{2} \\
& =\frac{1}{t^{2} m^{2}}\left\|\left\{a_{n}\right\}_{n=1}^{m}\right\|_{\ell_{2}}^{2} \leq \frac{1}{t^{2} m}\left\|\left\{a_{n}\right\}\right\|_{\ell_{\infty}}^{2}
\end{aligned}
$$

Furthermore, for almost every $x \in[0,1]$,

$$
\max \left\{\frac{1}{m} \sum_{n=1}^{m} a_{n} r_{n}(x):\left|a_{n}\right| \leq 1\right\}=1
$$

For $m \in \mathbb{N}$ let $p_{m}=1+2+\cdots+m, p_{0}=0$. If $n, m \in \mathbb{N}$ and $p_{m-1}<n \leq$ $p_{m}$, let $s_{n}(x)=\frac{1}{m} r_{n-p_{m-1}}\left(2^{m}\left(x-2^{-m}\right)\right)$ for $x \in\left[\frac{1}{2^{m}}, \frac{1}{2^{m-1}}\right]$ and $s_{n}(x)=0$ otherwise. Fix $\left\{a_{n}\right\} \in \ell_{\infty}$ and let $f=\sum_{n=1}^{\infty} a_{n} s_{n}$. Note that $f(0)=0$. The function $f$ is approximately continuous at 0 since for every $t>0$ and every $m \in \mathbb{N}$,

$$
\lambda\left(\left\{x \in\left[\frac{1}{2^{m}}, \frac{1}{2^{m-1}}\right]:|f(x)|>t\right\}\right) \leq \frac{1}{2^{m}} \frac{1}{t^{2} m}\left\|\left\{a_{n}\right\}\right\|_{\ell_{\infty}}^{2} .
$$

We have also

$$
\max \left\{\sum_{p_{m-1}<n \leq p_{m}} a_{n} s_{n}(x):\left|a_{n}\right| \leq 1\right\}=1
$$


for almost all $x \in\left[\frac{1}{2^{m}}, \frac{1}{2^{m-1}}\right]$. Now, for $n, m \in \mathbb{N}, p_{m-1}<n \leq p_{m}$, let $\widetilde{s}_{n}$ be a continuous function on $\mathbb{R}$, supported in the same interval as $s_{n},\left\|\widetilde{s}_{n}\right\| \leq \frac{1}{m}$, such that $\lambda\left(\left\{x \in \mathbb{R}: \widetilde{s}_{n}(x) \neq s_{n}(x)\right\}\right) \leq \frac{1}{m^{2} 2^{m}}$. Let $T_{1}: \ell_{\infty} \rightarrow \mathcal{A}$ be defined by $T_{1}\left(\left(\alpha_{1}, \beta_{1}, \alpha_{2}, \beta_{2}, \ldots\right)\right)=\sum\left(\alpha_{n}-\beta_{n}\right) \widetilde{s}_{n}$. Then obviously $\left\|T_{1}\right\| \leq 2$.

In order to prove non-regularity of $T_{1}$, assume that there exists a positive operator $P: \ell_{\infty} \rightarrow \mathcal{A}, P \geq T_{1}$. Let $M_{k}, k \in \mathbb{N}$, be disjoint and infinite subsets of $\mathbb{N}, N_{k}=\left\{n: p_{m-1}<n \leq p_{m}\right.$ for some $\left.m \in M_{k}\right\}$. Set $b_{2 n-1}^{(k)}=b_{2 n}^{(k)}=1$ if $n \in N_{k}$, zero otherwise. Fix $k \in \mathbb{N}$ and let $f_{k}=P\left(\left\{b_{n}^{(k)}\right\}_{n=1}^{\infty}\right) \in \mathcal{A}$. Then, for every $m \in M_{k}$,

$$
f_{k}(x) \geq \max \left\{\sum_{p_{m-1}<n \leq p_{m}}\left(\alpha_{n}-\beta_{n}\right) \widetilde{s}_{n}(x): 0 \leq \alpha_{n} \leq 1,0 \leq \beta_{n} \leq 1\right\}=1
$$

for all $x \in\left[\frac{1}{2^{m}}, \frac{1}{2^{m-1}}\right] \backslash A_{m}$ where $A_{m}$ is a set of measure at most $\frac{1}{m 2^{m}}$. Thus $f_{k}(0) \geq 1$ since $f_{k}$ is approximately continuous. Let $c_{n}=1$ for all $n \in \mathbb{N}$. By positivity and linearity of $P, P\left(\left\{c_{n}\right\}\right)(0) \geq P\left(\left\{\sum_{k=1}^{K} b_{n}^{(k)}\right\}\right)(0) \geq K$ for every $K \in \mathbb{N}$, which is a contradiction.

Corollary 6. There exists a continuous operator $T_{2}: \mathcal{A} \rightarrow \mathcal{A}$ which is not regular.

Proof. Note that if $M=\left\{x_{n}: n \in \mathbb{N}\right\} \subset \mathbb{R}$ is infinite and scattered (and $x_{n} \neq x_{m}$ for $n \neq m$ ) then the map $\iota:\left.B_{1}\right|_{M} \rightarrow \ell_{\infty}: f \mapsto\left\{f\left(x_{n}\right)\right\}_{n=1}^{\infty}$ is an order preserving isometry $\left.B_{1}\right|_{M}$ onto $\ell_{\infty}$ (since every function on $M$ extended by zero on $\mathbb{R} \backslash M$ is a Baire one function by the same argument as in the proof of Lemma 2). Therefore we will not distinguish between these two spaces.

For $f \in \mathcal{A}$, let $T_{2}(f)=T_{1}(r(f))$ where $T_{1}: \ell_{\infty} \rightarrow \mathcal{A}$ is the continuous non-regular operator from the previous example and $r$ is the restriction map to an infinite scattered set $M \subset \mathbb{R}\left(\right.$ e.g. $M=\mathbb{N}$ ). Let $L:\left.\mathcal{B}_{1}\right|_{M} \rightarrow \mathcal{A}$ be a positive simultaneous extension operator (Theorem 4 ). Then $T_{2}$ is not regular since otherwise $T_{1}=T_{1} r L=T_{2} L$ were regular, too.

Now let $M \subset \mathbb{R}$ be an infinite set of Lebesgue measure zero. Let $M_{1} \subset M$ be an infinite scattered set (e.g. a sequence converging to $x \in \mathbb{R} \cup\{ \pm \infty\}$ ) and $M_{2}=M \backslash M_{1}$. Then, since $f=f \cdot \chi_{M_{1}}+f \cdot\left(1-\chi_{M_{1}}\right)$ for every $f \in \mathcal{B}_{1}$ and $\chi_{M_{1}} \in \mathcal{B}_{1},\left.\mathcal{B}_{1}\right|_{M}$ is isometrically and order isomorphic to $\left.\left.\mathcal{B}_{1}\right|_{M_{1}} \oplus_{\infty} \mathcal{B}_{1}\right|_{M_{2}}$ and hence to $\left.\ell_{\infty} \oplus_{\infty} \mathcal{B}_{1}\right|_{M_{2}}$. If $T_{1}$ is as in Example 5, then $T_{3}((x, f))=T_{1}(x)$ $\left(x \in \ell_{\infty},\left.f \in \mathcal{B}_{1}\right|_{M_{2}}\right)$ provides a continuous non-regular operator $\left.\mathcal{B}_{1}\right|_{M} \rightarrow \mathcal{A}$. Furthermore, assume we have a continuous simultaneous extension operator $L:\left.\mathcal{B}_{1}\right|_{M} \rightarrow \mathcal{A}$. Then it can be easily modified (using the above ideas) to a non-regular one. Thus the idea of simplification of the proof of Theorem 4 using the notion of regular operator fails. 
Finally, we would like to note that it is not difficult to show directly that any of conditions (iii), (iv), (v) of Theorem 4 implies that $M$ is countable.

\section{References}

[J] G. J. O. Jameson, An elementary proof of the Arens and Borsuk extension theorems, J. London Math. Soc. II. Ser. 14 (1976), 364-368.

[K] K. Kuratowski, Topology I, Academic Press 1966.

[LMZ] J. Lukeš, J. Malý, L. Zajíček, Fine Topology Methods in Real Analysis and Potential Theory, Lecture Notes in Mathematics 1189, SpringerVerlag 1986.

[PL] G. Petruska, M. Laczkovich, Baire 1 functions, approximately continuous functions and derivatives, Acta Math. Acad. Sci. Hung. 25 (1974), 189-212.

[X] Hong-Yun Xiong, On whether or not $\mathcal{L}(E, F)=\mathcal{L}^{r}(E, F)$ for some classical Banach lattices $E$ and $F$, Indagationes Math. 46 (1984), 267282. 